\title{
Semiconductor microcavities for enhanced nonlinear optics interactions
}

\author{
Alessio Andronico \\ Xavier Caillet \\ Ivan Favero \\ Sara Ducci \\ Vincent Berger \\ Giuseppe Leo \\ giuseppe.leo@univ-paris-diderot.fr
}

\begin{abstract}
Laboratoire "Matériaux et Phénomènes Quantiques", CNRS-UMR 7162 Université Paris Diderot Case courrier 7021, 75205, Paris Cedex 13, France

Laboratoire "Matériaux et Phénomènes Quantiques", CNRS-UMR 7162 Université Paris Diderot Case courrier 7021, 75205, Paris Cedex 13, France

Laboratoire "Matériaux et Phénomènes Quantiques", CNRS-UMR 7162 Université Paris Diderot Case courrier 7021, 75205, Paris Cedex 13, France

Laboratoire "Matériaux et Phénomènes Quantiques", CNRS-UMR 7162 Université Paris Diderot Case courrier 7021, 75205, Paris Cedex 13, France

Laboratoire "Matériaux et Phénomènes Quantiques", CNRS-UMR 7162 Université Paris Diderot Case courrier 7021, 75205, Paris Cedex 13, France

Laboratoire "Matériaux et Phénomènes Quantiques", CNRS-UMR 7162 Université Paris Diderot Case courrier 7021, 75205, Paris Cedex 13, France
\end{abstract}

Semiconductor microcavities offer a unique way to enhance nonlinear optical processes through light confinement in space and time. In this article we review two different nonlinear optics semiconductor-based applications that benefit from the microcavity setting. Firstly, we discuss a difference frequency generation scheme in a GaAs microdisk. Secondly, we show how a recently demonstrated source of counter-propagating twin photons can display a sensible performance improvement when combined with a vertical cavity. [DOI: $10.2971 /$ jeos.2008.08030]

Keywords: nonlinear optics, microcavities

\section{INTRODUCTION}

Since the birth of nonlinear optics, optical resonators have been regarded as a promising way to enhance nonlinear interactions. This is simply due to the fact that a strong energy buildup in small volumes can significantly reduce the threshold needed to trigger nonlinear phenomena. With the development of nanoscale fabrication techniques, it is now possible to confine photons in structured microcavities of a few cubic microns, and store them for a considerable amount of time. In these devices, the spatial confinement is typically quantified by the mode volume $V_{M}$, whereas the temporal confinement is described by the quality factor $Q$, which relates the energy $E$ stored in the cavity to the energy $\Delta E$ lost in a cycle: $Q=2 \pi E / \Delta E$.

For these reasons, a considerable amount of research was devoted to the study and optimization of small volume and high- $Q$ microcavities. High quality photonic crystal $(\mathrm{PhC})$ resonators, for example, have proved to be very effective in obtaining ultra-low-power optical bistable switching, SecondHarmonic Generation (SHG), and in modifying the nonlinear susceptibility of materials through the Purcell effect [1].

Another interesting approach relies on Whispering Gallery Mode (WGM) resonators such as microspheres [2] and microdisks [3]. In this case the light is guided by the bent dielectric/air interface and, by interfering constructively with itself at resonance, gives rise to an intensity build-up. This geometry allows to reach a very high storage time: e. g. quality factor as high as $Q=5 \times 10^{6}$ and $Q=3.6 \times 10^{5}$ have been reported at telecom wavelengths in $\mathrm{Si}$ [4] and AlGaAs [5] microdisks, respectively.

In this paper, we discuss two microcavity-based nonlinear optics applications: 1) a Difference Frequency Generation (DFG) between phase-matched WGMs in a GaAs microdisk; 2) the optimization of a recently demonstrated source of counterpropagating twin photons [6].

In both cases, the semiconductor technology is a key factor in terms of compactness and integration potential.

\section{WGMS MICROCAVITIES}

WGM resonators are well known optoelectronic components and their use as semiconductor lasers [3] or add-drop filters [7] is well established. In nonlinear optics, $\chi^{(3)}$ processes have also been extensively studied in dielectric materials: for example, ultralow-threshold Raman laser using a silica microsphere [8], or Kerr induced parametric oscillation [9] in a silica microtoroid.

Conversely, the exploitation of $\chi^{(2)}$ interactions between 
WGMs has been much less explored so far. The first WGMbased SHG proposal was done in 2003 by Ilchenko and coworkers [10]. They subsequently demonstrated their device using a toroidal PPLN cavity [11] and, more recently, obtained non-degenerate parametric oscillation in an unpoled $\mathrm{LiNbO}_{3}$ disk [12].

In this context, GaAs microdisks promise peculiar advantages like: a mature semiconductor technology for a fully optoelectronic integration; a large refractive index for strong field confinement; and a huge nonlinear coefficient that can be exploited to obtain high efficiencies. To date, the only drawback in the choice of GaAs was its optical isotropy, but this problem can be overcome following the theoretical analysis reported in $[13,14]$. In those works, the authors show that the symmetry of a [100]-grown AlGaAs microdisk results in a periodic modulation of the effective nonlinear coefficient experienced by nonlinearly interacting WGMs. Specifically, they address a SHG process, where this modulation is used to obtain a hybrid modal/quasi-phase-matching scheme. The key issue of this approach is that it does not rely on material periodic domain inversion. The same approach, combined with two bus waveguides side-coupled to an AlGaAs microring, also promises to enhance the Spontaneous Parametric Down Conversion (SPDC) in order to generate spatially separated entangled photons [15].

Here we extend these analyses to the case of a DFG scheme in the wavelength range between 2.5 and $2.9 \mu \mathrm{m}$, as a possible alternative to GaInAsSb/AlGaAsSb quantum-well lasers for room-temperature, continuous-wave spectroscopic applications [16]. In this section, after reviewing the linear and nonlinear properties of WGMs resonators, we design a triplyresonant GaAs microdisk with optimized performances (Section 2.3).

\subsection{Linear Theory of WGMS Resonators}

Let us briefly outline the method that we use to find the resonance frequencies of a microdisk cavity similar to the one showed in Figure 1.

In our model, the vertical structure is taken into account

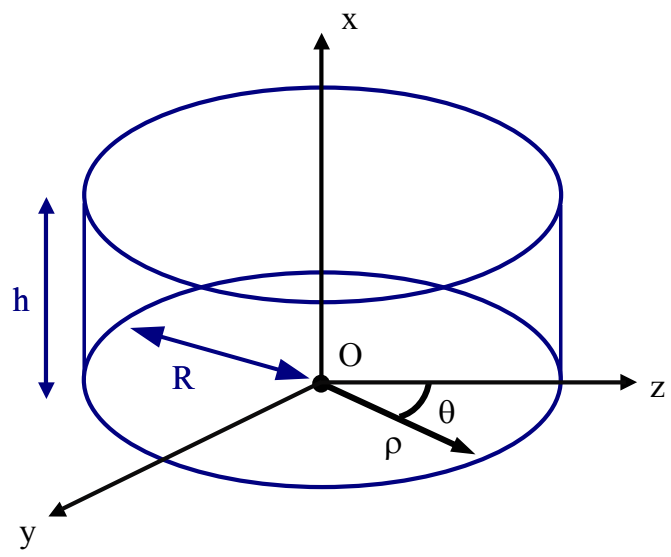

FIG. 1 Microdisk of radius $R$ and thickness $h$. The cylindrical reference system used in the text is shown. through the Effective Index Method (EIM): we first find the effective index of the vertical structure $\left(n_{\alpha}\right.$ with $\alpha=$ TE/TM) and then replace the $3 D$ structure with a simpler $2 D$ disk whose refractive index equals the effective index found in the previous step. By doing this, it is straightforward to verify that Maxwell's equations split into two sets of uncoupled equations for TE and TM modes. For a TM mode, the electric field is polarized orthogonal to the microdisk plane and the only non-zero magnetic field components are $H_{\rho}$ and $H_{\theta}$. For a TE mode, conversely, the magnetic field only possesses the $x$ component whereas the electric field lies in the plane of the cavity. In both cases the wave equation is simply:

$$
\frac{d^{2} \psi}{d \rho^{2}}+\frac{1}{\rho} \frac{d \psi}{d \rho}+\left(n^{2} \tilde{k}^{2}-\frac{m^{2}}{\rho^{2}}\right) \psi=0,
$$

where $n=n_{\alpha}$ inside the microdisk and $n=1$ outside, $\tilde{k}$ is the wavenumber, the integer $m$ is the azimuthal number (i.e. the number of wavelengths that fits the circumference) and $\psi=E_{x}\left(\psi=H_{x}\right)$ for TM (TE) modes.

Eq. (1) can be solved in terms of Bessel $\left(J_{m}\right)$ and Hankel $\left(H_{m}^{(2)}\right)$ functions of order $m$ :

$$
\psi= \begin{cases}N J_{m}\left(\tilde{k} n_{\alpha} \rho\right) e^{-j m \theta+j \tilde{\omega} t} & \rho \leq R \\ N B H_{m}^{(2)}(\tilde{k} \rho) e^{-j m \theta+j \tilde{\omega} t} & \rho>R,\end{cases}
$$

where $N$ is a normalization constant, $R$ is the microdisk radius, $\tilde{\omega}=\tilde{k} c$, and $B=J_{m}\left(\tilde{k} n_{\alpha} R\right) / H_{m}^{(2)}(\tilde{k} R)$ [17].

The continuity of tangential $\mathbf{E}$ and $\mathbf{H}$ results in the dispersion relations:

$$
\begin{gathered}
n_{T M} \frac{\dot{J}_{m}\left(\tilde{k} n_{T M} R\right)}{J_{m}\left(\tilde{k} n_{T M} R\right)}-\frac{\dot{H}_{m}^{(2)}(\tilde{k} R)}{H_{m}^{(2)}(\tilde{k} R)}=0 \quad \text { (TM modes) } \\
\frac{\dot{J}_{m}\left(\tilde{k} n_{T E} R\right)}{J_{m}\left(\tilde{k} n_{T E} R\right)}-n_{T E} \frac{\dot{H}_{m}^{(2)}(\tilde{k} R)}{H_{m}^{(2)}(\tilde{k} R)}=0 \quad \text { (TE modes). }
\end{gathered}
$$

Eqs. (3) and (4) can be numerically solved to find the resonance eigenfrequencies $\tilde{\omega}$ of the cavity: once that the wavelength and the azimuthal order $m$ are fixed, different resonances can be found, corresponding to radial order $p$. The lowest frequency mode corresponds to $p=0$, whereas higher frequency modes have higher $p$ values.

It is worth noting that, despite the formal analogy with the optical slab waveguide case, the eigenfrequencies obtained here are complex numbers. This results from the curved device geometry: since the disk has a bent boundary, all its resonances are affected by radiation losses. These losses can be quantified by defining the resonance quality factor of a mode as $Q_{W G M}=\operatorname{Re}(\tilde{\omega}) /(2 \operatorname{Im}(\tilde{\omega}))$.

In conclusion, the resonances of a microdisk can be labeled by using three "quantum" numbers: the vertical order $q$, which accounts for the vertical confinement ${ }^{1}$; the azimuthal order $m$, which expresses the mode rotational symmetry; and the radial order $p$, which accounts for the mode radial profile.

\footnotetext{
${ }^{1}$ Throughout the rest of the paper we will always consider the fundamental vertical mode, that is $q=0$.
} 


\subsection{Nonlinear Frequency Mixing Between WGMS}

If the microdisk material possesses sufficiently high $\chi^{(2)}$, two pump modes injected into the cavity can generate another mode at the difference frequency. This DFG scheme is illustrated in Figure 2: two pump fields, with frequencies corresponding to two microdisk's eigenfrequencies ( $\omega_{1}$ and $\left.\omega_{2}\right)$, are injected in a tapered fiber evanescently coupled to the cavity [18]. There, the nonlinear polarization is a source for a third mode at $\omega_{3}=\omega_{1}-\omega_{2}$; if this mode corresponds to a microdisk resonance, an efficient frequency mixing process is possible.

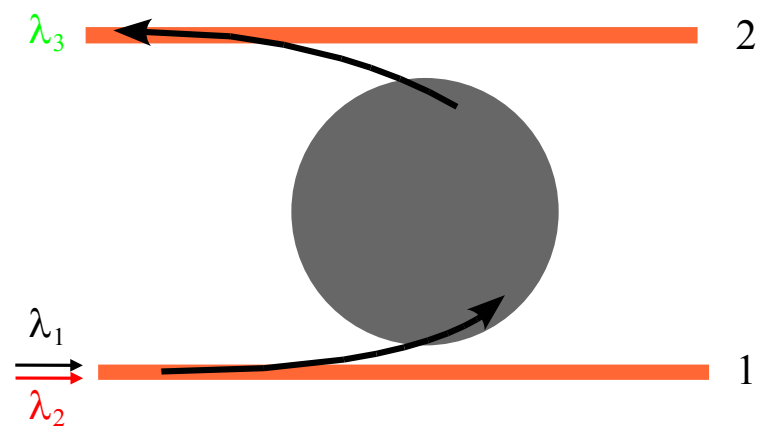

FIG. 2 Coupling scheme for the DFG process. Two pump fields are injected through fiber 1 , whose distance to the disk is optimized to maximize the coupling. Fiber 2 allows an efficient extraction of the generated mode.

Using standard Coupled Mode Theory (CMT), we can write simple equations describing the nonlinear process [19]:

$$
\begin{aligned}
& \frac{d a_{1}}{d t}=j \omega_{1} a_{1}-\frac{a_{1}}{\tau_{1}^{t o t}}+\sqrt{\frac{2}{\tau_{1}^{c p l}}} s_{1}+s_{1}^{N L} \\
& \frac{d a_{2}}{d t}=j \omega_{2} a_{2}-\frac{a_{2}}{\tau_{2}^{t o t}}+\sqrt{\frac{2}{\tau_{2}^{c p l}}} s_{2}+s_{2}^{N L} \\
& \frac{d a_{3}}{d t}=j \omega_{3} a_{3}-\frac{a_{3}}{\tau_{3}^{t o t}}+s_{3}^{N L} .
\end{aligned}
$$

For the $i$ th resonant mode $(i=1,2,3), a_{i}$ is the mode amplitude normalized to the energy, $\tau_{i}^{\text {tot }}=2 Q_{i}^{\text {tot }} / \omega_{i}$ is the photon lifetime (including intrinsic and coupling losses) and $s_{i}^{N L}$ is the nonlinear source term normalized to the power. Moreover, in the first two equations, the terms $s_{1}$ and $s_{2}$ describe the sourcing from fiber 1 , and are normalized to the power.

In the following, we will put ourselves in the undepleted pump approximation by neglecting $s_{1}^{N L}$ and $s_{2}^{N L}$. In this way, by putting $a_{i}=A_{i} e^{j \omega_{i} t}(i=1,2)$ and looking for the steady state solution of the first two equations in (5), we find:

$$
\left|A_{i}\right|^{2}=\frac{4}{\omega_{i}} \frac{Q_{i}^{c p l}}{\left(1+Q_{i}^{c p l} / Q_{i}^{i n t}\right)^{2}} P_{i}^{i n} \quad(\text { for } \mathrm{i}=1,2),
$$

where $P_{i}^{i n}$ is the fiber input power, $Q_{i}^{c p l}$ is the loss term due to the presence of the coupling to the fiber, $Q_{i}^{i n t}$ accounts for all the intrinsic loss mechanisms, and $1 / Q^{t o t}=1 / Q^{i n t}+1 / Q^{c p l}$. In particular, $Q_{i}^{\text {int }}$ has contributions from: bending losses (what we previously defined as $Q_{W G M}$ ), material absorption losses, nonlinear (intensity dependent) absorption losses, surface losses etc. From Eq. (6) we see that the transfer of power from the fiber to the cavity is maximized when the condition $Q_{i}^{c p l}=Q_{i}^{i n t}$ is fulfilled (critical coupling). This condition can be achieved by properly adjusting the distance fiber-resonator.

On the other hand, the last of Eqs. (5) still contains the term $s_{3}^{N L}$, which is given by [19]:

$$
\begin{aligned}
s_{3}^{N L}= & -\frac{j \omega_{3}}{4} \epsilon_{0} a_{1} a_{2}^{*} \\
& \times \int_{V} \sum_{i j k} \chi_{i j k}^{(2)}\left(\omega_{3} ; \omega_{1},-\omega_{2}\right) E_{i}^{*}\left(\omega_{3}\right) E_{j}\left(\omega_{1}\right) E_{k}^{*}\left(\omega_{2}\right) d V \\
= & -\frac{j \omega_{3}}{4} \epsilon_{0} a_{1} a_{2}^{*} I_{o v l},
\end{aligned}
$$

with $V$ the cavity volume. This equation expresses the fact that the nonlinear source term responsible of the intra-cavity generation of the mode at $\omega_{3}$ is essentially an overlap integral between the three resonant modes multiplied by the $\chi^{(2)}$ tensor.

Since GaAs has the zincblende structure with $\overline{4} 3 m$ symmetry [20] and we are considering the growth axis oriented in the [100] direction, the overlap integral and the nonlinear polarization differ from zero in two cases: 1) two TE pump modes and a TM output; 2) two orthogonally polarized pump modes and a TE output. The argument of the overlap integral in Eq. (7) can be written as:

$$
d_{14}\left\{\bar{C}_{+} e^{j\left(m_{2}+m_{3}-m_{1}+2\right) \theta}+\bar{C}_{-} e^{j\left(m_{2}+m_{3}-m_{1}-2\right) \theta}\right\},
$$

where $\bar{C}_{+}$and $\bar{C}_{-}$depend on the profiles of the modes involved in the DFG. Then it is clear that the source term $s_{3}^{N L}$ is always zero unless the following conditions are fulfilled:

$$
\begin{gathered}
\omega_{3}=\omega_{1}-\omega_{2} \quad \text { (Energy Conservation), } \\
\Delta m=m_{2}+m_{3}-m_{1} \pm 2=0 \quad \text { (Momentum Conservation) }
\end{gathered}
$$

These conditions generalize what was found in $[13,14]$ for the case of a SHG process: the \pm 2 is due to the additional momentum provided by the periodic modulation of the $\chi^{(2)}$ tensor that results from the circular geometry.

Returning to Eqs. (5) and taking $a_{3}=A_{3} e^{-j \omega_{3} t}$, we readily find the steady-state solution:

$$
\frac{A_{3}}{\tau_{3}^{t o t}}=-\frac{j \omega_{3}}{4} \epsilon_{0} A_{1} A_{2}^{*} I_{o v l}
$$

Since the power at $\omega_{3}$ coupled to fiber 2 is given by $P_{3}^{\text {out }}=$ $\left|\sqrt{2 / \tau_{3}^{c p l}} A_{3}\right|^{2}$, the energy circulating inside the cavity is related to the incident power through Eq. (6) and under the hypothesis of critical coupling for both the pumps and the DF mode, we have:

$$
P_{3}^{\text {out }}=\frac{1}{16} Q_{1}^{\text {int }} Q_{2}^{\text {int }} Q_{3}^{\text {int }} \frac{\omega_{3}}{\omega_{1} \omega_{2}} \epsilon_{0}^{2}\left|I_{o v}\right|^{2} P_{1}^{\text {in }} P_{2}^{\text {in }},
$$

which gives the conversion efficiency:

$$
\eta=\frac{P_{3}^{\text {out }}}{P_{1}^{\text {in }}}=\frac{1}{16} Q_{1}^{\text {int }} Q_{2}^{\text {int }} Q_{3}^{\text {int }} \frac{\omega_{3}}{\omega_{1} \omega_{2}} \epsilon_{0}^{2}\left|I_{o v}\right|^{2} P_{2}^{\text {in }} .
$$

Therefore, at critical coupling, the conversion efficiency is directly related to the time that the WGMs spend within the cavity: a higher $Q$-factor results in a longer interaction time between the fields involved in the nonlinear mixing. 


\subsection{Results and Discussion}

In order to generate light in the wavelength band $2.5-2.9 \mu \mathrm{m}$, we have designed a GaAs disk with radius $R=3.7 \mu \mathrm{m}$ and thickness $h=1.8 \mu \mathrm{m}$. By doing so, two DFG interactions can be phase matched: 1) with two TE pump modes at $\lambda_{1}=0.983 \mu \mathrm{m}$ and $\lambda_{2}=1.567 \mu \mathrm{m}$, and a TM output at $\left.\lambda_{3}=2.639 \mu \mathrm{m} ; 2\right)$ with a TE pump mode at $\lambda_{1}=0.973 \mu \mathrm{m}$, a TM pump mode at $\lambda_{2}=1.533 \mu \mathrm{m}$, and a TE output at $\lambda_{3}=2.658 \mu \mathrm{m}$.

As suggested above, the two inputs and the output of the microdisk are evanescently coupled to two optical fibers, which fulfill the critical-coupling condition. Under such hypothesis, for fiber input powers of $1 \mathrm{~mW}$ and $Q^{\text {int }}=3 \times 10^{4}$, the predicted efficiencies are $\eta_{1}=4.6 \times 10^{-4}$ and $\eta_{2}=4.4 \times 10^{-4}$. These values depend on the nonlinear overlap integral of the three interacting modes, which is related to the radial mode profiles as shown in Eq. (7). An example of these profiles is shown in Figure 3 for the case of two TE pump modes and a TM output.

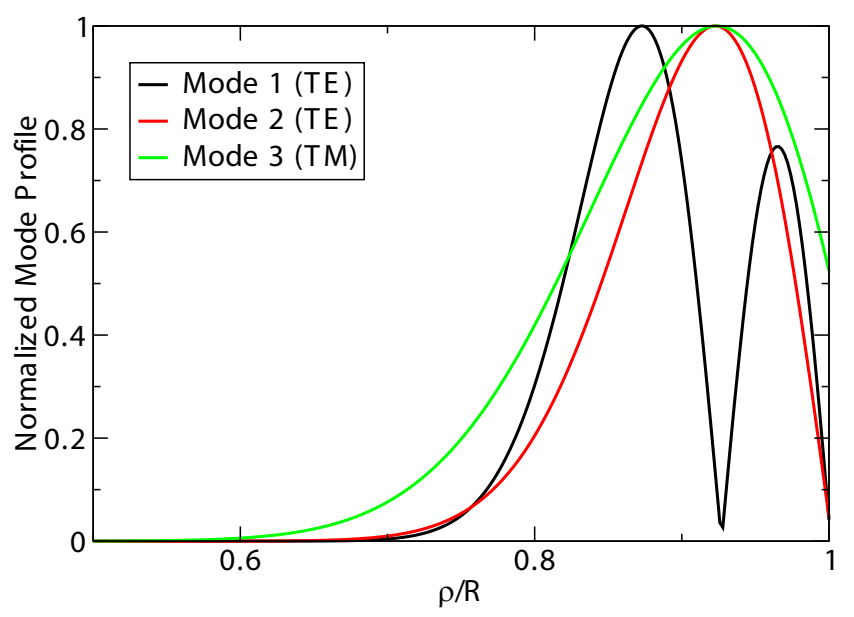

FIG. 3 Profiles of the WGMs involved in the first DFG described in the text. The two pump fields are TE polarized, whereas the intra-cavity generated mode is TM polarized.

By properly adjusting the microdisk radius and thickness, triplets of phase-matched wavelengths could be found, which nicely cover a DFG wavelength range from $\lambda_{3}=2.5 \mu \mathrm{m}$ to $\lambda_{3}=2.9 \mu \mathrm{m}$. With a view to a feasible experiment, this DFG process could be obtained with pump wavelength ranges $\lambda_{1} \in$ $[0.98,0.99] \mu \mathrm{m}$ and $\lambda_{2} \in[1.56,1.57] \mu \mathrm{m}$, readily available from a Ti:Sapphire and an external-cavity telecom semiconductor laser, respectively.

Clearly, the wavelength of the generated mode depends on the disk fabrication tolerances $( \pm 10 \mathrm{~nm}$ on both $R$ and $t)$. In order to illustrate this point, we numerically explored the dependence of the phase-matched triplet of wavelengths on radius variations around $R=3.7 \mu \mathrm{m}$ (with fixed $h=1.8 \mu \mathrm{m}$ ), and reported such dependence in Figure 4 for $\lambda_{3}$ and $\lambda_{1}$. Here it is shown that a small error on the microdisk radius only produces a slight shift of the phase-matched WGM wavelengths.

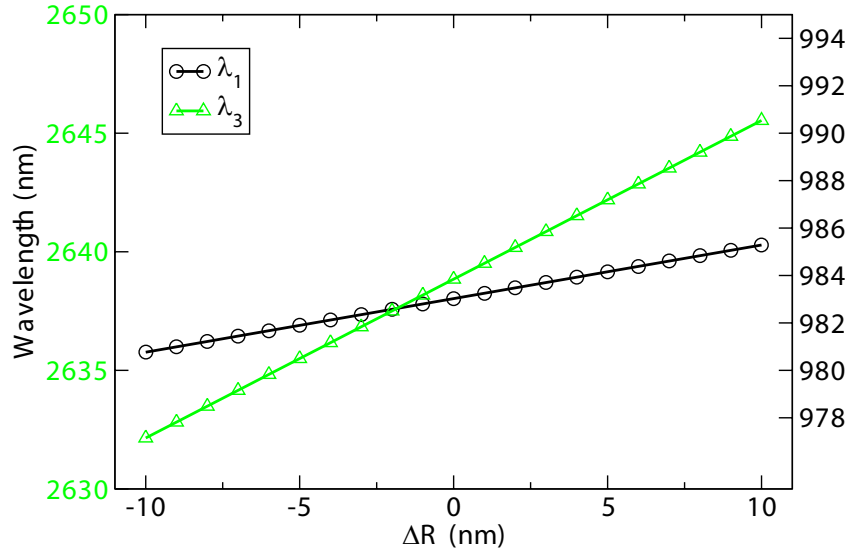

FIG. 4 Dependence of the pump $\left(\lambda_{1}\right)$ and intra-cavity generated mode wavelength $\left(\lambda_{3}\right)$ on small radius variations around the nominal value $R_{0}=3.7 \mu \mathrm{m}$. The conversion efficiency is nearly the same $\left(\eta \approx 5 \times 10^{-4}\right)$ in the whole tolerance interval.

\section{RIDGE MICROCAVITIES}

Semiconductor microcavities based on Distributed Bragg Reflectors (DBR) have been extensively studied in the past years due to their strong impact on applications and light matter interactions. Among the photonic devices based on these kind of cavities, we can cite VCSELs [21], light-emitting diodes and nonlinear optical switches. In the context of fundamental research, the strong coupling between exciton and photonic cavity mode is an intense field of study in this moment [22].

In all of these cases, light is strongly confined in one or, by additional lateral patterning, more dimensions, like in ridge microcavities (2D confinement) [23] or micropillars (3D confinement) [24].

The field enhancement induced by the cavity can also be used to increase the efficiency in three wave mixing processes such as SHG or SPDC. In this context we can mention several works concerning a "transverse pump configuration", where one of the interacting beams is parallel to the cavity axis and orthogonal to the other ones $[25,26]$. The first experimental demonstration of this kind of interactions has been an SHG experiment with a ridge microcavity [27].

It has also been proposed to use SPDC in the transverse pump configuration, in order to realize a counter-propagating twin photon source [28], and the corresponding experimental demonstration has been reported in [6]. Several advantages arise from this geometry: automatic separation of the downconverted photons, large tunability, narrow spectral bandwidth. Moreover, frequency correlated, anticorrelated or even uncorrelated two-photon states can be generated by an appropriate control of the spatial and spectral pump field properties [29].

Thereafter we first illustrate the working principle of the source; then we show the effect of adding a vertical microcavity on the conversion efficiency. 


\subsection{A source of counter-propagating twin photons: basic idea}

The twin-photon source presented here is a multilayer AlGaAs waveguide designed to allow a counter-propagating phase matching scheme. The lateral confinement is provided by a wet-etched ridge. In this geometry (Figure 5), a pump field $(780 \mathrm{~nm})$ impinges on top of the waveguide generating two counter-propagating, orthogonally polarized waveguided twin photons $(\approx 1560 \mathrm{~nm})$ through SPDC. The frequencies of the emitted fields are fixed by the energy $\left(\omega_{p}=\right.$ $\left.\omega_{s}+\omega_{i}\right)$ and momentum $\left(k_{p} \sin \theta=n_{s} k_{s}-n_{i} k_{i}\right)$ conservation, where $\omega_{p}, \omega_{s}$ and $\omega_{i}\left(k_{p}, k_{s}\right.$ and $\left.k_{i}\right)$ are the frequencies (wavevectors) of pump, idler and signal; $\theta$ is the angle of incidence of the pump beam, and $n_{s}$ and $n_{i}$ are the effective indices of the signal and idler modes.

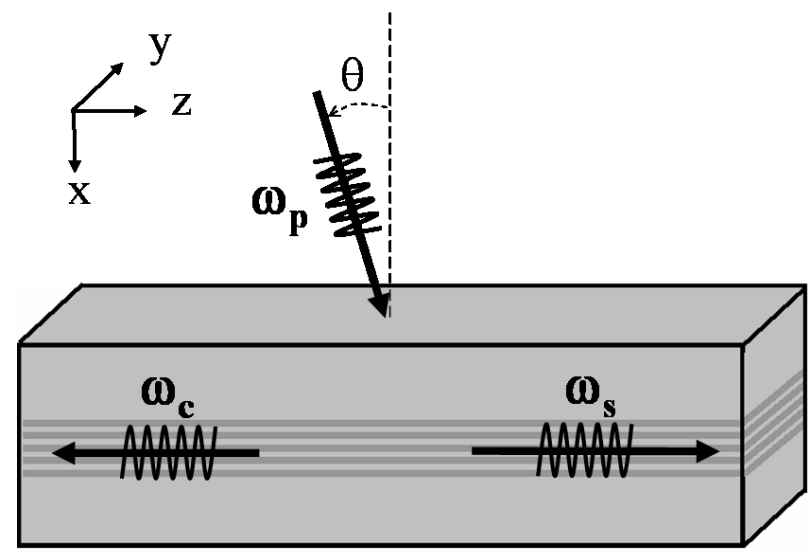

FIG. 5 Schematic of the presented semiconductor twin photon source. The generated signal and idler photons are guided, counter-propagating and orthogonally polarized. The pump beam is incident with an angle $\theta$. The longer the waveguide, the narrower the spectral bandwidth of the down-converted photons.

Momentum conservation in the epitaxial direction is satisfied by alternating $\mathrm{AlGaAs}$ layers with different $A l$ concentration.

The conversion efficiency of the source, defined as the number of photon pairs per pump photon, is [30]:

$$
\eta_{0}=\frac{N_{\text {pairs }}}{N_{p}}=\frac{2 \hbar \omega_{s} \omega_{i} \omega_{p}}{\epsilon_{0} c^{2} n_{s} n_{i}} \frac{0.886}{b} \frac{|\Gamma|^{2}}{W \cos \theta}
$$

where $W$ is the width of the waveguide, $b=1 / v_{g}^{(s)}+1 / v_{g}^{(i)}$ is the sum of the inverse group velocities of signal and idler modes, and $\Gamma$ is the overlap integral given by:

$$
\Gamma=\iint d_{e f f} E_{p}(x, y) E_{s}(x, y) E_{i}(x, y) d x d y,
$$

with $d_{e f f}$ the effective nonlinear coefficient. In this expression, $E_{s}, E_{i}, E_{p}$ are the signal, idler and pump electric fields, which are appropriately normalized. Several frequency conversion processes have been experimentally demonstrated with this source: SHG [30], DFG [31], and SPDC [6]. In the last case, a time-correlation experiment demonstrated the twin character of the generated photons. The measured conversion efficiency is very close to the theoretical value $\eta_{0}=1.5 \times 10^{-13}$ for a $3 \mu \mathrm{m}$ wide waveguide. This low value stems from the the unguided character of the pump.
An interesting possibility to improve the source performances consists in the addition of DBRs on top of and under the waveguide, in order to create a microcavity for the pump beam. In this case, at resonance wavelength, a nearly standing wave is obtained inside the cavity, whose amplitude can be largely greater than outside.

\subsection{Efficiency enhancement through the vertical microcavity}

In the case of the simple waveguide presented above, the guided field amplitude of the pump beam can be roughly estimated using the Fresnel equations. Assuming, for the sake of simplicity, a homogeneous waveguide with refractive index $n_{\text {guide }}$ and air as incident medium, the relation between the internal and external pump field is $E_{p}^{i n t}=t E_{p}^{e x t}$ with $|t|=2 n_{\text {Air }} /\left(n_{\text {Air }}+n_{\text {guide }}\right)$.

In the case of the ridge microcavity showed in Figure 6, we can use the transfer matrix method to compute the amplitude of the standing wave inside the cavity. This is found to be:

$$
\frac{E_{p}^{i n t}}{E_{p}^{e x t}}=2 \sqrt{\frac{2 n_{\text {Air }} F}{\pi n_{\text {guide }}\left(1+\left|t_{2} / t_{1}\right|\right)}} .
$$

Here, $F \equiv \frac{\pi \sqrt{R}}{1-R}$ is the finesse of the cavity, with $R=\left|r_{1} r_{2}\right| ; r_{1}$ and $r_{2}\left(t_{1}\right.$ and $\left.t_{2}\right)$ are the reflection (transmission) coefficients associated to the front $(i=1)$ and back $(i=2)$ mirrors. It can be shown that the conversion efficiency enhancement factor due to the addition of the microcavity is:

$$
\frac{\eta_{\text {cavity }}}{\eta_{0}}=\frac{2\left(n_{\text {Air }}+n_{\text {guide }}\right)^{2}}{\pi n_{\text {Air }} n_{\text {guide }}} \frac{F}{\left(1+\left|t_{2} / t_{1}\right|\right)} .
$$

This expression indicates how to optimize the conversion efficiency. Firstly, the ratio $\left|t_{2} / t_{1}\right|$ has to be minimized, reflecting the obvious fact that the front mirror should be less reflective

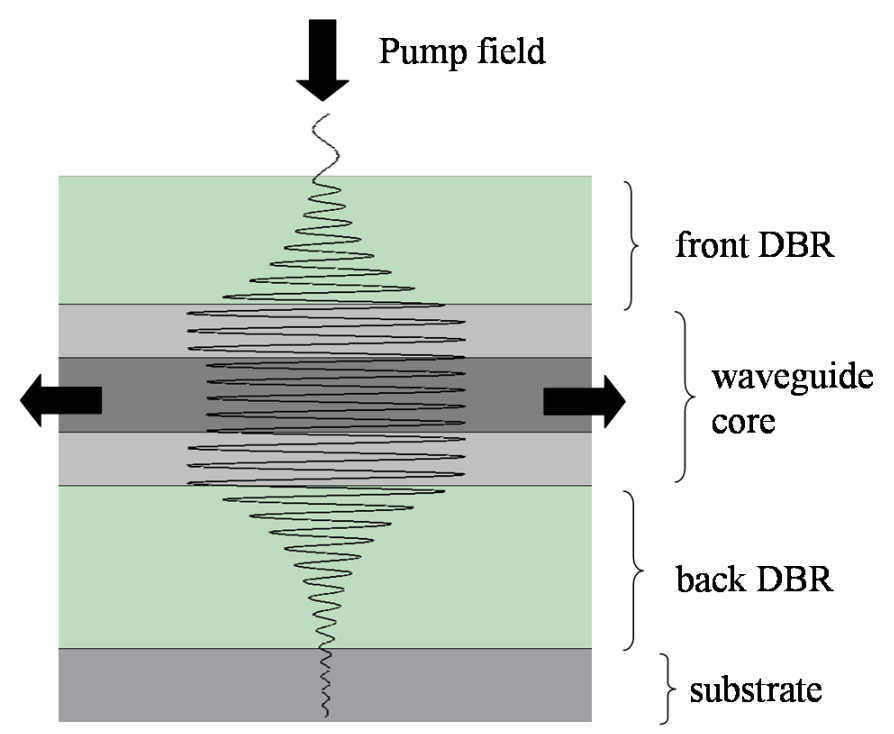

FIC. 6 View in the $(x z)$ plane of the waveguide with the addition of the DBRs. In black is plotted the pump beam profile within the structure at cavity resonance. The field enhancement induces a strong improvement of the conversion efficiency. 
than the back one, in order to let the light enter the microcavity. Secondly, a maximum finesse is desired. It is interesting, for the rest of the discussion, to introduce the quality factor of the cavity: $Q=m_{e f f} F$. Here, $m_{e f f}$ is the effective order of the cavity, given by $m_{e f f}=m+m_{0}$, where $m$ is the cavity order and $m_{0}$ accounts for the penetration of the field in the DBR. For a $\lambda / 4$ DBR stack, $m_{0}=n_{H} /\left(n_{H}-n_{L}\right)$, where $n_{H}$ and $n_{L}$ are the refractive indices of the high- and low-index materials. Eq. (13) shows that the higher the quality factor, the higher the efficiency.

However, a practical limitation on $Q$ stems from technical reasons. Firstly, the large amount of energy stored in the cavity can possibly induce thermorefractive effects, leading to undesirably bistable devices. Moreover, the inhomogeneity of the sample inherent to the growth process induces a variation of the cavity resonance wavelength $\lambda$ along the direction of the waveguide. To obtain an efficient interaction throughout the structure, this variation has to be smaller than the mean cavity linewidth $\delta \lambda$. This, in return, sets an upper limit to the desired quality factor, expressed as $Q=\frac{\lambda}{\delta \lambda}$.

\subsection{Discussion}

The previous analysis demonstrates that the quality factor should be limited to avoid spurious effects. In these conditions, aiming at a maximized finesse, and consequently maximized conversion efficiency, leads to the minimization of the effective order $m_{\text {eff }}$ of the cavity.

Alternative solutions are being presently envisaged and will be published elsewhere. Nevertheless, an estimation of the possible efficiency enhancement can be given: a reasonable linewidth of $\delta \lambda=0.5 \mathrm{~nm}$ and an effective cavity order $m_{e f f}=$ 10 , along with symmetric mirrors, gives a finesse $F=300$ and an efficiency enhancement factor: $\eta_{\text {cavity }} / \eta_{0}=500$.

\section{CONCLUSION}

In this paper, we discussed two nonlinear optical applications under the perspective of exploiting the microcavity field enhancement to increase the efficiency of a nonlinear parametric process. This step was accomplished by designing, in the first case, an high- $Q$ triply resonant microdisk for DFG and, in the second, an optimized ridge cavity for the generation of twinphotons. Both these applications benefit from the advantages provided by the choice of GaAs in terms of strong confinement, huge $\chi^{(2)}$ and mature semiconductor technology.

\section{ACKNOWLEDGEMENTS}

We gratefully acknowledge support from EU No. MRTN-CT2004-51240 POISE.

\section{References}

[1] J. Bravo-Abad, A. Rodriguez, P. Bermel, S. J. Johnson, J. D. Joannopoulos, and M. Soljačić, "Enhanced Nonlinear Optics in
Photonic-Crystal Microcavities" Opt. Express 15, 16161-16176 (2007).

[2] V. B. Braginsky, M. L. Gorodetsky, and V. S. Ilchenko, "QualityFactor and Nonlinear Properties of Optical Whispering-Gallery Modes" Phys. Lett. A 137, 393-397 (1989).

[3] S. L. McCall, A. F. J. Levi, R. E. Slusher, S. J. Pearton, and R. A. Logan, "Whispering-Gallery Mode Microdisk Lasers" Appl. Phys. Lett. 60, 289-291 (1992).

[4] M. Borselli, T. J. Johnson, and 0. Painter, "Beyond the Rayleigh Scattering Limit in High- $Q$ Silicon Microdisk: Theory and Experiment" Opt. Express 13, 1515-1530 (2005).

[5] K. Srinivasan, M. Borselli, T. J. Johnson, P. E. Barclay, 0. Painter, A. Stintz, and S. Krishna, "Optical Loss and Lasing Characteristics of High-Quality-Factor AlGaAs Microdisk Resonators with Embedded Quantum Dots" Appl. Phys. Lett. 86, 151106 (2005).

[6] L. Lanco, S. Ducci, J.-P. Likforman, X. Marcadet, J. A. W. van Houwelingen, H. Zbinden, G. Leo, and V. Berger, "Semiconductor Waveguide Source of Counterpropagating Twin Photons" Phys. Rev. Lett. 97, 173901 (2006).

[7] E. J. Klein, D. H. Geuzebroek, H. Kelderman, G. Sengo, N. Baker, and A. Driessen, "Reconfigurable Optical Add-Drop Multiplexer Using Microring Resonators" IEEE Photonic. Tech. L. 17, 2358-2360 (2005).

[8] S. M. Spillane, T. J. Kippenberg, and K. J. Vahala, "UltralowThreshold Raman Laser Using a Spherical Dielectric Microcavity" Nature 415, 621-623 (2002).

[9] T. J. Kippenberg, S. M. Spillane, and K. J. Vahala, “Kerr-Nonlinearity optical Parametric 0scillation in an Ultrahigh- $Q$ Toroid Microcavity" Phys. Rev. Lett. 93, 083904 (2004).

[10] V. S. Ilchenko, A. B. Matsko, A. A. Savchenkov, and L. Maleki, “LowThreshold Parametric Nonlinear Optics with Quasi-Phase-Matched Whispering-Gallery Modes" J. Opt. Soc. Am. B 20, 1304-1309 (2003).

[11] V. S. Ilchenko, A. A. Savchenkov, A. B. Matsko, and L. Maleki, "Nonlinear Optics and Crystalline Whispering Callery Mode Cavities" Phys. Rev. Lett. 92, 043903 (2004).

[12] A. A. Savchenkov, A. B. Matsko, M. Mohageg, D. V. Strekalov, and L. Maleki, "Parametric Oscillations in a Whispering Gallery Resonator" Opt. Lett. 32, 157-159 (2007).

[13] Y. Dumeige, and P. Féron, "Whispering-Gallery-Mode Analysis of Phase-Matched Doubly Resonant Second-Harmonic Ceneration" Phys. Rev. A 74, 063804 (2006).

[14] Z. Yang, P. Chak, A. D. Bristow, H. M. van Driel, R. Iyer, J. S. Aitchison, A. L. Smirl, and J. E. Sipe, "Enhanced Second-Harmonic Generation in AlGaAs Microring Resonators" 0pt. Lett. 32, 826-828 (2007).

[15] Z. Yang, and J. E. Sipe, "Cenerating Entangled Photons Via Enhanced Spontaneous Parametric Downconversion in AlGaAs Microring Resonators" Opt. Lett. 32, 3296-3298 (2007).

[16] C. Lin, M. Grau, 0. Dier, and M.-C. Amann, "Low Threshold Room-Temperature Continuous-Wave Operation of $2.24-3.04 \mu \mathrm{m}$ GalnAsSb/AlGaAsSb Quantum-Well Lasers" Appl. Phys. Lett. 84, 5088-5090 (2004).

[17] J. E. Heebner, T. C. Bond, and J. S. Kallman, "Ceneralized Formulation for Performance Degradations due to Bending and Edge Scattering Loss in Microdisk Resonators" Opt. Express 15, 4452-4473 (2007).

[18] J. C. Knight, G. Cheung, F. Jacques, and T. A. Birks, "Phase-Matched Excitation of Whispering-Gallery-Mode Resonances by a Fiber Taper" Opt. Lett. 22, 1129-1131 (1997).

[19] H. A. Haus, Waves and Fields in Optoelectronics (Prentice-Hall, 
Englewood Cliffs, 1984).

[20] A. Yariv, Quantum Electronics (Wiley, New York, 1984).

[21] K. Iga, F. Koyama, and S. Kinoshita, "Surface Emitting Semiconductor Lasers" IEEE J. Quantum. Elect. 24, 1845-1855 (1988).

[22] C. Weisbuch, M. Nishioka, A. Ishikawa, and Y. Arakawa, "Observation of the Coupled Exciton-Photon Mode Splitting in a Semiconductor Quantum Microcavity" Phys. Rev. Lett. 69, 3314-3317 (1992).

[23] A. Kuther, M. Bayer, T. Gutbrod, A. Forchel, P. A. Knipp, T. L. Reinecke, and R. Werner, "Confined Optical Modes in Photonic Wires" Phys. Rev. B 58, 15744-15748 (1998).

[24] J. M. Gérard, D. Barrier, J. Y. Marzin, R. Kuszelewicz, L. Manin, E. Costard, V. Thierry-Mieg, and T. Rivera, "Quantum Boxes as Active Probes for Photonic Microstructures: The Pillar Microcavity Case" Appl. Phys. Lett. 69, 449-451 (1996).

[25] R. Lodenkamper, M. M. Fejer, and J. S. J. Harris, "Surface Emitting Second Harmonic Generation in Vertical Resonator" Electron. Lett. 27, 1882-1884 (1991).

[26] G. Beadie, W. S. Rabinovich, and Y. J. Ding, “Transversely Pumped
Nonlinear Structure which Generates Counterpropagating Guided Waves: Theory and Numerical Modeling" IEEE J. Quantum. Elect. 37, 863-872 (2001).

[27] R. Lodenkamper, M. L. Bortz, M. M. Fejer, K. Bacher, and J. S. J. Harris, "Surface-Emitting Second-Harmonic Generation in Semiconductor Vertical Resonator" Opt. Lett. 18, 1798-1800 (1993).

[28] A. De Rossi, and V. Berger, "Counterpropagating Twin Photons by Parametric Fluorescence" Phys. Rev. Lett. 88, 043901 (2002).

[29] X. Caillet, V. Berger, G. Leo, and S. Ducci, “A Semiconductor Source of Counterpropagating Twin Photons: A Versatile Device Allowing the Control of the Two-Photon State" to be published in Modern Optics.

[30] M. Ravaro, Y. Seurin, S. Ducci, G. Leo, V. Berger, A. De Rossi, and G. Assanto, "Nonlinear AlGaAs Waveguide for the Generation of Counterpropagating Twin Photons in the Telecom Range" J. Appl. Phys. 98, 063103 (2005).

[31] L. Lanco, S. Ducci, J.-P. Likforman, M. Ravaro, P. Filloux, X. Marcadet, G. Leo, and V. Berger, "Backward Difference Frequency Generation in an AlGaAs Waveguide" Appl. Phys. Lett. 89, 031106 (2006). 